\title{
Solvent effects on extraction of polycyclic aromatic hydrocarbons in ambient aerosol samples
}

\author{
Mira Flasch ${ }^{1}$, Magdalena Kistler2,a, Bernadette Kirchsteiger ${ }^{1}$ and Anne Kasper-Giebl ${ }^{1}$ \\ ${ }^{1}$ Institute of Chemical Technologies and Analytics, Vienna University of Technology, Vienna, Austria
}

\begin{abstract}
Polycyclic Aromatic Hydrocarbons (PAHs) in the ambient particulate matter pose one of the most important issues in the focus of environmental management. The concentration of their representative, Benzo(a)pyrene (BaP), undergoes limitations according to European Union directive. However, a successful control over the pollution levels and their sources is limited by the high uncertainty of analytical and statistical approaches used for their characterization. Here we compare differences in PAH concentrations related to the use of different solvents in the course of ultrasonic extraction of a certified reference material (PM10-like PAH mixture) and filter samples of ambient particulate matter collected in Austria for the CG-MS PAH analysis. Using solvents of increasing polarity: Cyclohexane (0,006), Toluene $(0,099)$, Dichloromethane $(0,309)$, Acetone $(0,43)$ and Acetonitrile $(0,460)$, as well as mixtures of those, filters representing high and low concentrations of particulate matter were investigated. Although some scatter of the obtained concentrations was observed no trend related to the polarity of the solvent became visible. Regarding the reproducibility, which can be expected of PAH analysis no significant difference between the different solvents was determined. This result is valid for all compounds under investigation.
\end{abstract}

\section{Introduction}

Polycyclic aromatic hydrocarbons (PAHs) are organic compounds consisting of two or more condensed aromatic rings. They are ubiquitous in the atmosphere and they are burdened with different adverse health effects [2]. Thus they are known to be carcinogenic and mutagenic compounds [3, 4]. Consequently, emissions and ambient concentrations have to be controlled and monitored. Principally PAHs are formed during the incomplete combustion of organic matter, i.e. often by anthropogenic activities based on the combustion of biomass or fossil fuels. The European emission inventory (EMEP) states that emission densities of PAHs in Europe are strongly influenced by domestic heating [5], while earlier the main influence of industries and transportation was outlined [6].

Due to its high toxicity Benzo(a)pyrene ( $\mathrm{BaP})$ is most thoroughly studied. It often is used as an indicator for the overall concentration of PAHs in ambient air. A limit value for $\mathrm{BaP}$ was defined within the $\mathrm{EU}$. This value equals $1 \mathrm{ng} / \mathrm{m}^{3}$, to be determined in particulate matter samples with a size cut of $10 \mu \mathrm{m}$ a.d. (PM10) as an average value for one calendar year [1]. During the last years this limit value was exceeded at a number of stations in Europe [7]. Comprehensive investigations of PAH concentrations cover additional compounds as well. Besides their actual concentrations the toxicity of the other PAHs can be expressed by a 'benz(a)pyreneequivalent' (BaPE) [8]. The main $\mathrm{PAHs}$ required to derive

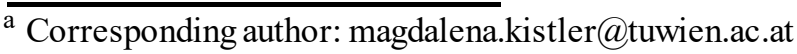

the $\mathrm{BaPE}$ can be found predominantly in the particulate phase [9].

To assure the conformity within the monitoring networks the analytical approach for $\mathrm{BaP}$ (and other PAHs) in the ambient aerosols is defined by the European Norm EN 15549 [10]. However, this document allows a choice of analytical approaches for the analysis of $\mathrm{BaP}$ (GC-MS or HPLC-FLD). This fact results in a relatively wide spectrum of the approved extraction methods and solvents. In this contribution we present the results of an experiment comparing the effects related to the use of different solvents in the course of ultrasonic extraction of filter samples for the CG-MS PAH analysis.

\section{Experimental}

\subsection{Sample preparation}

For the comparison two filter samples collected in Austria in winter 2010/11 were used. Ambient particulate matter was loaded onto quartz fibre filters, using High-Volume samplers. Sampling time was 24 hours and the sampled air volume was approximately $720 \mathrm{~m}^{3}$. To account for different filter loadings one sample was collected in an urban area (i.e. the city of Klagenfurt, Carinthia), representing a highly loaded filter (Filter 1). The second sample (Filter 2) was loaded in a rural region (Arnfels, Styria) and represents conditions typical for lower particulate matter concentrations. Furthermore, a standard 
reference material (1649a, Urban Dust) was used for the intercomparison.

To obtain equally loaded sub-samples, the filters were cut into sixteen segments of equal area using a special cutting tool. For each analyses one of these segments was used. The filter segments were placed in glass Petri dishes. For the determination of the extraction recovery the samples were spiked with a deuterated standard $\left(\mathrm{d}_{12-}\right.$ perylene). Afterwards the filter segments were transferred to a glass test tube. For analysis of the reference material approximately $150 \mathrm{mg}$ of the material was directly weighed into the test tube and spiked there. The respective solvents were added to a total volume of $5 \mathrm{~mL}$. Five different solvents in seven different combinations were investigated. The applied solvent combinations are displayed in Table 1.

Table 1. Solvents and solvent mixtures used for the sample extraction

\begin{tabular}{|c|c|c|c|}
\hline Name & Solvent $\mathbf{1}[\mathbf{m L}]$ & $\begin{array}{c}\text { Solvent 2 } \\
{[\mathbf{m L}]}\end{array}$ & Ratio \\
\hline DCM & Dichloromethane & - & - \\
\hline DcH1 & Dicloromethane & Cyclohexane & $3: 2$ \\
\hline DcH2 & Dicloromethane & Cyclohexane & $3: 7$ \\
\hline cHex & Cyclohexane & - & - \\
\hline AcH & Acetone & Cyclohexane & $1: 1$ \\
\hline AcN & Acetonitrile & - & - \\
\hline TocH & Toluene & Cyclohexane & $3: 3$ \\
\hline
\end{tabular}

Subsequently the samples were put in an ultrasonic bath to ensure thorough extraction of the filters. The extraction time was $30 \mathrm{~min}$. The procedure was repeated once and the combined extracts were then spiked with an internal standard (d12-BaP). In a next step the extracts were evaporated to about $200 \mu l$ in a nitrogen flow. Only the samples extracted with acetonitrile were evaporated to dryness, then dissolved in $3 \mathrm{~mL}$ cyclohexane and put in an ultrasonic bath for $30 \mathrm{~min}$ before finally reducing the liquid volume to approx. $200 \mu \mathrm{l}$. As solids (washed off insoluble particulate matter or fibres of the quartz filters) could be present, especially in the extracts of the samples of the reference material, the extracts were centrifuged and just the clear supernatant was used for analysis.

\subsection{Analytical methods}

The PAHs determination was performed with a GC-MS method previously used by Kistler et al. [11]. The HP5890 Gas Chromatograph is equipped with a HP-7683 auto sampler and a split/split-less injector $\left(300^{\circ} \mathrm{C}\right)$ operated in a split-less mode ( $2 \mathrm{~min})$. A deactivated fused silica precolumn $(1 \mathrm{~m} \times 0.32 \mathrm{~mm})$ was used in combination with an analytical capillary column (DB-5 MS- 95\% dimethyl, $5 \%$ phenylsiloxane, $30 \mathrm{~m} \times 0.25 \mathrm{~mm}$ ID x $0.25 \mu \mathrm{m}$ film thickness) for the separation. The temperature programme started at $50^{\circ} \mathrm{C}$ for $2 \mathrm{~min}$, followed by an increase of the temperature to $150^{\circ} \mathrm{C}$ with a rate of $20^{\circ} \mathrm{C} \mathrm{min}{ }^{-1}$. Afterward the temperature was increased to $300^{\circ} \mathrm{C}$, with a rate of $10^{\circ} \mathrm{C} \mathrm{min}-1$ and the final temperature was held for $20 \mathrm{~min}$. The temperature was $230^{\circ} \mathrm{C}$ for the source, $150^{\circ} \mathrm{C}$ for the quadrupole and $300^{\circ} \mathrm{C}$ for the transfer line. Helium 5.0 (Messer) with a flow rate of $1 \mathrm{~mL} \mathrm{~min}^{-1}$ was used as a carrier gas. A quadruple mass spectrometer HP$5973(70 \mathrm{eV})$ was applied as a detector. PAHs were recorded in the full scan mode and selected ion monitoring (SIM) mode.

Standards were prepared using a mix-standard (PAKMix 18, Neochema) containing 18 PAHs to identify and quantify the compounds. Naphthalene, 2methylnaphthalene, 1-methylnaphthalene, acenaphthene, acenaphthylene, phenanthrene, fluorine, anthracene, benz[a]anthracene (BaA), chrysene (Chr), pyrene (PY), fluoranthene (FLT), benzo[b]fluoranthene (BbF), benzo[k]fluoranthene $(\mathrm{BkF})$, benzo[a]pyrene $(\mathrm{BaP})$, indeno[1,2,3-cd]pyrene (IcdP), benzo[ghi]perylene (BghiP) and dibenz[a,h]anthracene (DahA) were included in the mix-standard. However, PAHs lighter than pyrene were not quantified. Samples and standards were measured twice each time.

\section{Results and Discussion}

The results of the analysis of filter 1 (highly loaded filter) and filter 2 (light loaded filter) are summarized in Tables 2 and 3, respectively. Loadings of the respective PAHs are given as ng per $\mathrm{mm}^{2}$ of filter area to highlight differences in filter loadings. Table 4 lists the results obtained for the reference material. Standard deviations given for the single compounds and measurement were calculated based on the variations obtained for the multiple injection $(n=5)$ of the $\mathrm{DcH} 2$ extract. Additionally, the mean value, determined for all extractions was calculated and listed in the last column. To assign an overall uncertainty to that average we use variations, more precisely the expanded uncertainties for the single PAHs and concentration levels, determined within an inter-laboratory intercomparison of $\mathrm{PAH}$ analyses on quartz filters [12]. Consequently, the variations given for the mean do not reflect standard variations of our measurements, but an estimate for an expanded uncertainty which can be expected for analysis using various methods for the determination of those compound. In case of filter 1 and the standard reference material the overall expanded uncertainties determined for higher concentration levels (F30 - as given in [12]) for the respective compounds were applied, while the overall expanded uncertainty determined for lower concentrations (F10 - as given in [12]) was used for filter 2 . Values of the single analyses falling out of the overall expanded uncertainty of the mean are marked in bold print. 
Table 2. Results of the determination of PAHs on filter 1 with different solvents including standard deviations of the measurement (calculated as given in the text)

\begin{tabular}{|c|c|c|c|c|c|c|c|c|}
\hline Analyte $\left[\mathrm{ng} / \mathrm{mm}^{2}\right]$ & DCM & DcH1 & DcH2 & cHex & AcH & $\mathbf{A c N}$ & TocH & Mean \\
\hline PY & $30.3 \pm 1.4$ & $40.0 \pm 1.9$ & $32.7 \pm 1.5$ & $26.7 \pm 1.2$ & $48.3 \pm 2.3$ & $37.6 \pm 1.8$ & $31.7 \pm 1.5$ & $35.3 \pm 9.3$ \\
\hline FLT & $28.7 \pm 1.9$ & $37.0 \pm 2.5$ & $30.1 \pm 2.0$ & $24.2 \pm 1.6$ & $47.5 \pm 3.2$ & $35.4 \pm 2.4$ & $27.5 \pm 1.9$ & $32.9 \pm 7.4$ \\
\hline $\mathrm{BaA}+\mathrm{Chr}$ & $41.0 \pm 0.9$ & $56.5 \pm 1.2$ & $53.9 \pm 1.1$ & $35.1 \pm 0.7$ & $47.5 \pm 1.0$ & $51.3 \pm 1.1$ & $44.4 \pm 0.9$ & $47.1 \pm 14.9$ \\
\hline $\mathrm{BbF}+\mathrm{BkF}$ & $60.0 \pm 2.6$ & $77.5 \pm 3.4$ & $70.2 \pm 3.0$ & $56.7 \pm 2.5$ & $58.1 \pm 2.5$ & $71.5 \pm 3.1$ & $36.9 \pm 1.6$ & $61.5 \pm 19.3$ \\
\hline $\mathrm{BaP}$ & $45.0 \pm 6.9$ & $56.5 \pm 8.7$ & $49.7 \pm 7.6$ & $39.5 \pm 6.1$ & $34.2 \pm 5.2$ & $54.7 \pm 8.4$ & $50.5 \pm 7.8$ & $46.6 \pm 13.8$ \\
\hline IcdP & $48.4 \pm 5.7$ & $55.0 \pm 6.5$ & $53.8 \pm 6.3$ & $39.5 \pm 4.6$ & $56.3 \pm 6.6$ & $53.0 \pm 6.2$ & $77.2 \pm 9.1$ & $54.7 \pm 20.3$ \\
\hline BghiP & $79.9 \pm 15.9$ & $96.3 \pm 19.2$ & $88.9 \pm 17.7$ & $61.3 \pm 12.2$ & $72.3 \pm 14.4$ & $95.2 \pm 19.0$ & $87.3 \pm 17.4$ & $83.0 \pm 33.7$ \\
\hline DahA & $15.6 \pm 0.8$ & $16.7 \pm 0.9$ & $15.5 \pm 0.8$ & $14.2 \pm 0.8$ & $12.1 \pm 0.6$ & $18.1 \pm 1.0$ & $15.7 \pm 0.8$ & $15.4 \pm 5.1$ \\
\hline
\end{tabular}

Table 3. Results of the determination of PAHs on filter 2 with different solvents including standard deviations of the measurement (calculated as given in the text)

\begin{tabular}{|c|c|c|c|c|c|c|c|c|}
\hline Analyte [ng/mm²] & DCM & DcH1 & DcH2 & cHex & AcH & $\mathbf{A c N}$ & TocH & Mean \\
\hline PY & $5.8 \pm 0.3$ & $7.3 \pm 0.3$ & $7.3 \pm 0.3$ & $7.1 \pm 0.3$ & $12.1 \pm 0.6$ & $6.7 \pm 0.3$ & $5.8 \pm 0.3$ & $7.4 \pm 2.7$ \\
\hline FLT & $5.8 \pm 0.4$ & $6.4 \pm 0.4$ & $7.1 \pm 0.5$ & $6.0 \pm 0.4$ & $11.5 \pm 0.8$ & $6.2 \pm 0.4$ & $5.2 \pm 0.4$ & $6.9 \pm 2.8$ \\
\hline $\mathrm{BaA}+\mathrm{Chr}$ & $2.8 \pm 0.1$ & $3.6 \pm 0.1$ & $3.5 \pm 0.1$ & $3.3 \pm 0.1$ & $4.1 \pm 0.1$ & $3.5 \pm 0.1$ & $2.9 \pm 0.1$ & $3.4 \pm 1.5$ \\
\hline $\mathrm{BbF}+\mathrm{BkF}$ & $4.1 \pm 0.2$ & $5.7 \pm 0.2$ & $5.8 \pm 0.3$ & $4.5 \pm 0.2$ & $6.4 \pm 0.3$ & $5.5 \pm 0.3$ & $6.2 \pm 0.3$ & $5.9 \pm 1.8$ \\
\hline $\mathrm{BaP}$ & $4.3 \pm 0.7$ & $4.3 \pm 0.7$ & $4.5 \pm 0.7$ & $4.2 \pm 0.6$ & $4.7 \pm 0.7$ & $4.5 \pm 0.7$ & $4.7 \pm 0.7$ & $4.4 \pm 2.2$ \\
\hline IcdP & $6.8 \pm 0.8$ & $8.4 \pm 1.0$ & $7.1 \pm 0.8$ & $6.4 \pm 0.7$ & $8.4 \pm 1.0$ & $8.5 \pm 1.0$ & $7.5 \pm 0.9$ & $7.6 \pm 2.9$ \\
\hline BghiP & $5.7 \pm 1.1$ & $6.5 \pm 1.3$ & $6.1 \pm 1.2$ & $4.7 \pm 0.9$ & $7.3 \pm 1.5$ & $8.3 \pm 1.7$ & $6.5 \pm 1.3$ & $6.4 \pm 2.8$ \\
\hline DahA & $4.1 \pm 0.2$ & $5.5 \pm 0.3$ & $4.5 \pm 0.2$ & $4.2 \pm 0.2$ & $5.5 \pm 0.3$ & $5.0 \pm 0.3$ & $4.5 \pm 0.2$ & $4.8 \pm 2.7$ \\
\hline
\end{tabular}

Table 4. Results of the determination of PAHs within the reference material with different solvents including standard deviations of the measurement (calculated as given in the text)

\begin{tabular}{|c|c|c|c|c|c|c|c|c|}
\hline Analyte [ng/mg] & DCM & DcH1 & DcH2 & cHex & AcH & AcN & TocH & Mean \\
\hline PY & $6.6 \pm 0.3$ & $4.0 \pm 0.2$ & $4.1 \pm 0.2$ & $3.4 \pm 0.2$ & $6.5 \pm 0.3$ & $5.4 \pm 0.3$ & $2.5 \pm 0.1$ & $5.0 \pm 1.3$ \\
\hline FLT & $6.8 \pm 0.5$ & $7.5 \pm 0.5$ & $7.7 \pm 0.5$ & $\mathbf{3 . 8} \pm \mathbf{0 . 3}$ & $\mathbf{1 0 . 0} \pm \mathbf{0 . 7}$ & $9.6 \pm 0.7$ & $5.6 \pm 0.3$ & $7.3 \pm 1.6$ \\
\hline BaA+Chr & $2.6 \pm 0.1$ & $2.0 \pm 0.1$ & $2.0 \pm 0.1$ & $1.4 \pm 0.1$ & $3.3 \pm 0.1$ & $2.8 \pm 0.1$ & $1.4 \pm 0.1$ & $2.2 \pm 0.7$ \\
\hline BbF+BkF & $3.8 \pm 0.2$ & $3.2 \pm 0.1$ & $2.8 \pm 0.1$ & $\mathbf{1 . 0} \pm \mathbf{0 . 1}$ & $\mathbf{5 . 1} \pm \mathbf{0 . 2}$ & $3.8 \pm 0.2$ & $2.0 \pm 0.1$ & $3.1 \pm 1.0$ \\
\hline BaP & $1.0 \pm 0.2$ & $0.7 \pm 0.1$ & $0.8 \pm 0.1$ & $0.4 \pm 0.1$ & $1.0 \pm 0.2$ & $0.6 \pm 0.1$ & $0.5 \pm 0.1$ & $0.7 \pm 0.2$ \\
\hline IcdP & $2.1 \pm 0.2$ & $2.3 \pm 0.3$ & $1.7 \pm 0.2$ & $\mathbf{0 . 9} \pm \mathbf{0 . 1}$ & $2.4 \pm 0.3$ & $1.7 \pm 0.2$ & $1.3 \pm 0.2$ & $1.8 \pm 0.7$ \\
\hline BghiP & $5.6 \pm 1.1$ & $5.1 \pm 1.0$ & $3.4 \pm 0.7$ & $\mathbf{0 . 9} \pm \mathbf{0 . 2}$ & $4.9 \pm 1.0$ & $2.8 \pm 0.6$ & $2.5 \pm 0.6$ & $3.5 \pm 1.5$ \\
\hline DahA & $0.9 \pm 0.1$ & $0.9 \pm 0.1$ & $0.7 \pm 0.1$ & $0.4 \pm 0.1$ & $0.9 \pm 0.1$ & $0.8 \pm 0.1$ & $0.6 \pm 0.1$ & $0.7 \pm 0.3$ \\
\hline
\end{tabular}

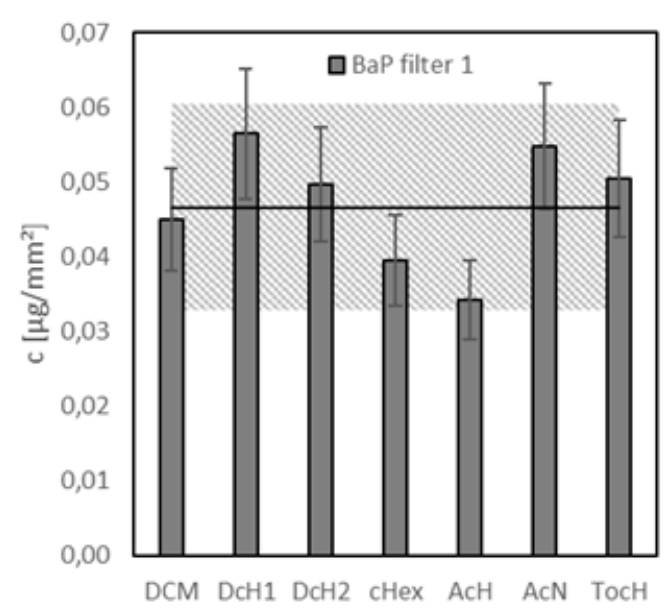

Figure 1. Filter loadings of BaP on filter 1, extracted with various solvents. Error bars reflect the standard deviation calculated by multiple injections of a single extract. The solid line represents the mean of all extractions, while the shaded area reflects the expanded uncertainty of the mean (calculated as given in the text).

Figures 1, 2 and 3 highlight the results exemplarily for $\mathrm{BaP}$. The solid line represents the mean value calculated based on all extracts, while the shaded area describes the expanded uncertainty obtained within an independent inter-laboratory intercomparison [12] and is explained in detail above.

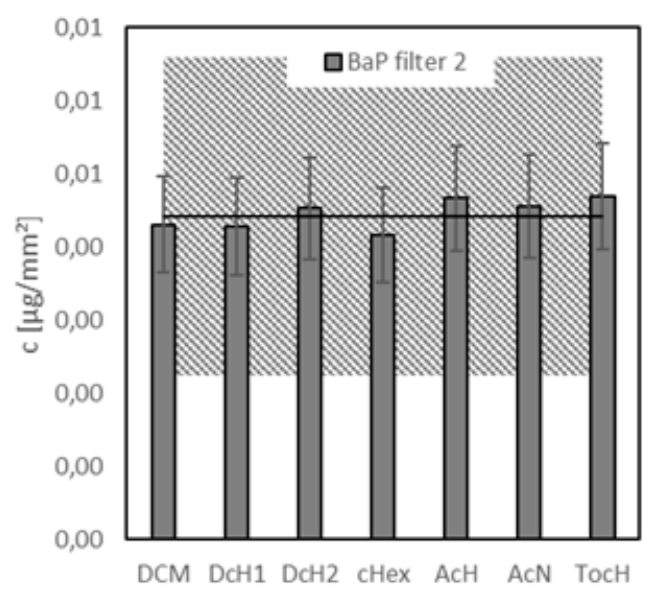

Figure 2. Concentration of $\mathrm{BaP}$ on filter 2, extracted with various solvents. Error bars reflect the standard deviation calculated by multiple injections of a single extract. The solid line represents the mean of all extractions, while the shaded area reflects the expanded uncertainty of the mean (calculated as given in the text). 
Although some scatter of the $\mathrm{BaP}$ concentrations determined with the respective solvents used for extraction is visible for the highly loaded filter and the reference material, all results remain within the overall expanded uncertainty of the mean. Very good agreement of the results, however, was obtained for filter 2 . In this case the variations of the filter loadings are within $7.5 \%$, even lower than the variations of multiple injections.

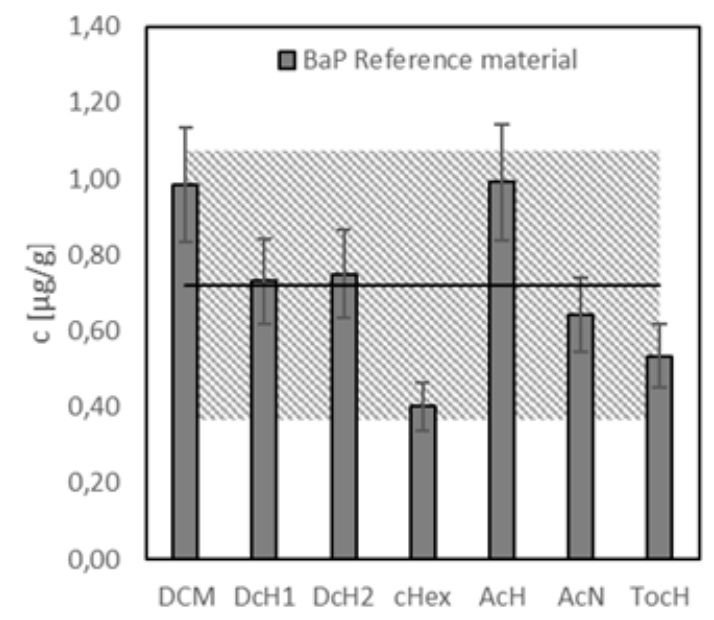

Figure 3. Concentration of $\mathrm{BaP}$ in the standard reference material, extracted with various solvents. Error bars reflect the standard deviation calculated by multiple injections of a single extract. The solid line represents the mean of all extractions, while the shaded area reflects the expanded uncertainty of the mean (calculated as given in the text).

The results obtained for the other PAHs are comparable to the findings shown for $\mathrm{BaP}$ and filter 1 . The excellent agreement obtained for $\mathrm{BaP}$ loadings on filter 2 extracted with the respective solvents was not seen for the other compounds. Still some variations of the measurements cannot be explained by the overall expanded uncertainty only. Using $\mathrm{TocH}$ as solvent $\mathrm{BbF}+\mathrm{BkF}$ (filter 1) fall below the expanded uncertainty area. In AcH FLT (filter 1+2, reference substance) and $\mathrm{BbF}+\mathrm{BkF}$ (reference substance) exceed the expanded uncertainty range. A more general trend was observed when using cHex as a solvent. In this case the underestimation of FLT, BbF+BkF, IcdP and BghiP in the reference material cannot be explained with the overall expanded uncertainty. Analyses of the filter samples do not clearly reflect or disprove this trend. Further investigations are needed to investigate this questions.

Overall the results indicate that the used solvents do not influence the extraction efficiency as much as expected.

\section{Acknowledgement}

Filter samples investigated here were collected within the EU project PMInter (www.pminter.eu).

\section{References}

1. Directive 2004/107/EC of the European Parliament and of the Council of 15 December 2004 relating to arsenic, cadmium, mercury, nickel and polycyclic aromatic hydrocarbons in ambient air, Official Journal of the European Union, L23/3-16 (2005)

2. T. Rengarajan, P. Rajendran, N. Nandakumar, B. Lokeshkumar, P. Rajendran, I. Nishigaki, Asian Pac. J. of Trop. Biomed. 5, 182-189 (2015)

3. A.O. Asita, M. Matsui, T. Nohmi, A. Matsuoka, M. Hayashi, M. Ishidate Jr., T. Sofuni, M. Koyano, H. Matsushita, Mutat. Res. Lett. 264,7-14 (1991)

4. P.H. Danielsen, S. Loft, A. Kocbach, P.E. Schwarze, P. Møller, Mutat. Res.-Genet. Tox. 674, 116-122 (2009)

5. EEA/LRTRAP, Air pollutant emissions data viewer (LRTAP Convention), Available online: http://www.eea.europa.eu/data-and-maps/data/dataviewers/air-emissions-viewer-lrtap (accessed on April, 2016)

6. Working Group on PAH, Ambient Air Pollution byPolycyclic Aromatic Hydrocarbons (PAH) Position Paper, Office for Official Publications of the European Communities, Luxembourg, ISBN 92-89-2057 (2001)

7. R. Gehring, Polyzyklische aromatische Kohlenwasserstoffe im PM10 an ausgewählten Stationen des NABEL sowie kantonalen Stationen, measurement report 2010, EMPA report Nr. 203056/17, CH 8600 Dübendorf

8. EPA, Provisional Guidance for Quantitative Risk Assessment of Polycyclic Aromatic Hydrocarbons, Office of Research and Development Washington, DC 20460, EPA/600/R-93/089 (1993)

9. K. Hytönen, P. Yli-Pirilä, J. Tissari, A. Gröhn, I. Riipinen, K.E.J. Lehtinen, J. Jokiniemi, Aerosol Sci. Tech. 43, 442-454

10. EN 15549:2008

11. M. Kistler, Particulate matter and odour emission factors from small scale biomass combustion units, $\mathrm{PhD}$, Vienna University of Technology (2012)

12. E. Grandesso, K. Kowaleswski, P.P. Ballesta, First EC-JRC PAHs inter-laboratory comparison on PM10 quartz filters, JRC Scientific and Technical Reports (2012) 